\title{
Physical Conditions of Cull Sows Associated with On-Farm Production Records
}

\author{
Mark Knauer $^{1^{*}}$, Ken Stalder ${ }^{2}$, Tom Baas ${ }^{2}$, Colin Johnson ${ }^{2}$, Locke Karriker ${ }^{3}$ \\ ${ }^{1}$ Department of Animal Science, North Carolina State University, Raleigh, USA \\ ${ }^{2}$ Department of Animal Science, Iowa State University, Ames, USA \\ ${ }^{3}$ Veterinary Diagnostic and Production Animal Medicine Department, Ames, USA \\ Email: ${ }^{*}$ mtknauer@gmail.com, tgkim@chonnam.ac.kr
}

Received July 7, 2012; revised July 31, 2012; accepted August 7, 2012

\begin{abstract}
Cull sow physical conditions were associated with on-farm production records. Sows (923) within one integrated U.S. production system were evaluated at two harvest facilities. Physical conditions evaluated at harvest were analyzed by parity, culling code and production measures. Farm culling codes were categorized into poor body condition (BC), old age $(\mathrm{G})$, lameness $(\mathrm{L})$, other $(\mathrm{O})$, poor litter performance $(\mathrm{P})$ and reproductive failure $(\mathrm{R})$. Production measures included lifetime pigs born alive (LPBA), pigs born alive in the last litter (PBALL), pigs per sow per year (PSY) and weaning to culling interval. The $\mathrm{L}$ culling code had a greater $(\mathrm{P}<0.05)$ prevalence of cracked hooves when compared to the other five culling codes $(30.9 \%$ vs. $18.7 \%)$. Sows without front cracked hooves tended $(\mathrm{P}=0.07)$ to have greater PSY $(0.80)$ when compared to sows having front cracked hooves. Females without rear digital overgrowth had more $(\mathrm{P}<0.05)$ PBALL $(0.54)$ and tended $(\mathrm{P}=0.06)$ to have increased PSY $(0.80)$ when compared to sows with rear digital overgrowth. Sows without shoulder lesions had greater $(\mathrm{P}<0.05)$ LPBA $(2.01)$ when compared to sows with shoulder lesions. Regression coefficient estimates for percent lung lesion involvement were positive and tended $(\mathrm{P}<0.10)$ to be different from zero for LPBA $(0.06)$ and PSY $(0.03)$. Females with severe teeth wear tended $(\mathrm{P} \leq 0.10)$ to have fewer LPBA (1.36), PBALL (0.45) and had fewer $(\mathrm{P}<0.01)$ PSY $(1.10)$ when compared to sows without severe teeth wear. Sows culled for $\mathrm{BC}$ and $\mathrm{L}$ had lower $(\mathrm{P}<0.01)$ backfat when compared to sows from the other four culling codes $(1.83$ and $2.04 \mathrm{~cm}$ vs. 2.47 to $2.85 \mathrm{~cm}$, respectively). Multiple cull sow physical conditions evaluated at harvest had associations with on-farm reproductive measures.
\end{abstract}

Keywords: Cull; Productivity; Sow

\section{Introduction}

Poor sow lifetime productivity in commercial pork production systems can lead to economic inefficiency and animal well-being concerns [1]. In the United States, reported annual breeding female culling rates are near $50 \%[2,3]$.

Traditional culling studies are based on retrospective farm data as they are relatively easy and economical to obtain. Producers are typically limited to reporting one reason for culling individual sows by their record keeping software. Additionally, these reasons are typically based on external signs or indications and do not incorporate information from internal and external body systems evaluation. Culling may be the result of multiple factors. Evaluating cull sows at harvest offers researchers an opportunity to confirm farm data and further identify factors related to sow culling. However, few studies investigating reasons for sow culling in harvest plants have

${ }^{*}$ Corresponding author. been reported in the scientific literature $[4,5]$.

Post-mortem reproductive organ examination offer potential information concerning sow reproductive failure. Foot lesions, disease, body condition and other body system problems may contribute to sow culling from commercial pork operations. Therefore, information from harvest plants can be used as a diagnostic tool to evaluate these lesions and further understand why sows are culled. The objective of this study was to relate the cull sows' physical conditions at harvest with on-farm production records within an integrated US pork production system.

\section{Materials and Methods}

\subsection{Animals and Housing}

Housing and animal care information is reported to provide the reader with management information with respect to how animals were housed and fed leading up to their harvest. A total of 923 sows from 8 farms within a 
single large integrated US pork production system were evaluated. Gestation sows were housed individually with solid concrete flooring in the front half and concrete slats in the rear half of the pen. Throughout gestation females were fed a gestation diet according to body condition [6] and had ad libitum access to water. Estrous detection was carried out once daily using fence-line boar contact. Females were bred using AI at 12 and $36 \mathrm{~h}$ after first detected estrus. Farrowing stalls contained either cast iron or wire metal slatted flooring. Sows were provided ad libitum access to feed and water throughout lactation. Gestation and lactation diets were balanced to meet or exceed NRC requirements [7].

\subsection{On-Farm Data Collection}

The Data collected from production records included sow identification, parity, first conception date, first farrowing date, lifetime pigs born alive (summation of pigs born alive across parities), pigs born alive in the last litter (prior to culling), farrowing and weaning dates, culling date and culling code. Data derived from on-farm production records included pigs per litter (lifetime pigs born alive)/(number of parities in the herd), non-productive days per parity [(culling date - first litter conception date) - (number of parities in the herd $\times 114$ )]/(number of parities in the herd), weaning to culling interval (culling date - last weaning date) and pigs per sow per year [(lifetime pigs born alive)/(culling date - first litter conception date) $\times 365$ ]. First litter conception date was used to calculate non-productive days per parity instead of herd entry date because of the variation in entry date to first conception ( $32 \pm 47 \mathrm{~d})$.

Farm culling codes were categorized into body condition $(\mathrm{BC})$, old age $(\mathrm{G})$, lameness $(\mathrm{L})$, other $(\mathrm{O})$, poor litter performance $(\mathrm{P})$ and reproductive failure $(\mathrm{R})$. Body condition included the farm codes poor body condition and unthrifty. Old age contained the farm codes old age and parity. Lameness consisted of the farm codes downer, injury and lameness. The culling code $\mathrm{O}$ included cesarean section, not found, other illness, prolapse, sudden death and unknown. Poor litter performance was comprised of farrowing complications, low number born alive, low number weaned, mastitis, poor milking ability and retained pigs. Reproductive failure included the farm codes abortion, did not conceive, no heat and not in pig. All farms culled sows on a weekly basis. No gilts (parity 0 females) were included in the study.

\subsection{Harvest Data Collection}

The Harvest facilities were chosen to represent the range of sows typically sent to market in the US. Because the type of sows harvested by individual facilities is driven by varied purchasing criteria based on anticipated products from those facilities, we chose two different Mid western plants for data collection. Plant 1 ( $n=281$ sows) harvested a thinner type of sow and Plant $2(n=642$ sows) harvested a heavier conditioned sow. Based on body condition score, ultrasonic backfat and ultrasonic loin muscle area we achieved our goal of evaluating a wide variety of cull sows. Twelve trips, six to each harvest plant, were made. Feet, shoulders, reproductive tracts, lungs, teeth and body condition were visually evaluated on individual harvested sows.

Front and rear foot lesions were evaluated and recorded by a trained technician. The technician examined each foot for the following abnormalities: 1) claw and hoof wall cracks (cracked hooves) including side-wall lesions, white line and toe cracks [8]; 2) pad (heel) lesions [8]; 3) the presence of abscesses on any foot surface; 4) abnormal overgrowth conditions (digital overgrowth) as defined by upward or inward toe curvature or excessive hoof growth $(2 \mathrm{~cm}$ longer than a normal toe); and 5) missing dew claws.

Shoulder lesions were evaluated and assigned to one of the following categories "none", "abscess", "abrasion", or "open" lesions. Lesion score was classified as "none" if the skin appeared normal over the point of the shoulder, an "abscess" lesion score was assigned when an opened or closed abscess was present, an "abrasion" lesion score was designated when fibrous tissue nodules were evident at the point of the shoulder [5] and an "open" lesion score was designated when an open, draining or healing sore was apparent at the point of the shoulder [5].

Reproductive tracts were removed from the carcass by harvest plant personnel and visually inspected immediately by the research veterinarian. From the macroscopic ovary examination sows were classified as normal (corpora lutea, corpora hemorrhagica or mature follicles were present), cystic (multiple follicular cysts, $>1.5 \mathrm{~cm}$ in diameter without corpora lutea or corpora hemorrhagica) or acyclic (no corpora lutea, corpora hemorrhagica and small follicles covering less $<50 \%$ of ovary). Pregnancy status was determined and fetal tissues, if present, were classified as normal, decomposed or mummified.

The thoracic and abdominal cavities and organs were visually evaluated for lesions by the research veterinarian. The presence or absence of peritonitis, pleural adhesions and lung lesions were recorded. If lung lesions were present, a total lung involvement percentage estimate was made. Lesions other than those previously mentioned were noted when visual evidence was present.

Teeth were evaluated by a trained technician. Top and bottom teeth were counted and scored for severity of wear. The following three definitions were used to categorize teeth wear: Minimum, sharp points present on molars and incisors; moderate, points on molars and incisors worn but grooves between points still evident; 
severe, no points or grooves present on molars and incisors.

A National Swine Improvement Federation certified real-time ultrasound technician measured backfat, longissimus muscle area, and longissimus muscle depth from a cross sectional 10th rib image using an Aloka $500 \mathrm{~V}$ SSD ultrasound machine (Corometrics Medical Systems, Inc., Wallingford, CT). At both plants, ultrasound evaluations were performed ante mortem while sows were restrained in a chute prior to harvest.

Body condition score (BCS) was evaluated using a scale of 1 (thin) to 5 (fat) [6]. At plant 1, BCS was evaluated as sows were suspended from a gambrel with their backs facing the observer. At plant 2, BCS was evaluated just prior to stunning as the animal was in a standing position. Technicians evaluating BCS were consistent across plants and visits, and had extensive livestock evaluation experience.

All data were recorded and reported at the sow level. In this study, attributing "left," "right" or "bilateral" characteristics to lesions in paired organs did not provide additional information as to cause or source and was not reliably obtained given the carcass fabrication process. Additionally, for the present study we did not find value in determining side or bilateral characteristics.

\subsection{Statistical Analysis}

The Statistical analyses were carried out using SAS [9]. Harvest facility data and on-farm production data were analyzed by parity and culling code. For the models comparing culling codes, parity was not included. Because so few culled sows had produced 10 or more parities, records from parity 10 and greater $(n=67)$ were combined into a single classification (10).

The GLIMMIX procedure can analyze response variables with a non-normal distribution while incorporating random effects into the model. Therefore, PROC GLIMMIX was used to analyze binary traits by parity and culling code. All models included the evaluator of the trait as a random effect.

The PROC MIXED procedure was used to analyze teeth number and body composition data by parity, culling code and body condition score. All models included the evaluator of the trait as a random effect. Parity was included as a fixed effect when analyzing backfat, longissimus muscle area and longissimus muscle depth by culling code and BCS.

The PROC MIXED procedure was used to analyze lifetime pigs born alive, pigs per litter, pigs born alive in the last litter, non-productive days per parity, weaning to culling interval and pigs per sow per year mean by parity and culling code. The models for parity and culling code included farm as a fixed effect.

\section{Results}

\subsection{Feet Lesions}

Physical characteristics and production LS MEANS by parity are shown in Table 1. The probability for front and rear heel lesion presence was different $(\mathrm{P}<0.01)$ between parities. Both front and rear heel lesions generally increased as parity increased. Front heel lesions ranged from $11.0 \%$ in parity 2 to $48.8 \%$ in parity 9 and rear heel lesions varied from $46.7 \%$ in parity 1 to $75.8 \%$ in parity 7. Physical characteristics and production LS MEANS by culling code are shown in Table 2. Front heel lesions were different $(\mathrm{P}<0.01)$ between culling codes. Old age had the greatest likelihood for front heel lesion presence (49.2\%). Physical characteristic contrasts (binary traits) and solution estimates (for continuous traits) for sow productivity traits are shown in Table 3. The weaning to culling interval for sows with no front heel lesions was longer $(\mathrm{P}<0.01)$ when compared to those with front heal lesions $(9.9 \mathrm{~d})$.

The probability for cull sows having cracked front hooves was different across parity of the sows and was generally greater in younger parity females. Front cracked hooves ranged from $36.6 \%$ in parity 2 to $12.9 \%$ in parity 5. The likelihood for the presence of front cracked hooves found on cull sows differed $(\mathrm{P}<0.01)$ among the culling codes. Sows within the L culling code had a greater probability for the presence of cracked hooves $(\mathrm{P}<0.05)$ than the other 5 culling codes combined $(30.9 \%$ vs. $18.7 \%)$. Cull sows without cracked hooves on their front feet tended $(\mathrm{P}=0.07)$ to have more pigs per sow per year $(0.80)$ when compared to sows with cracked hooves on the front feet. Sows without front cracked hooves had a shorter $(\mathrm{P}<0.05)$ weaning to culling interval $(5.4 \mathrm{~d})$ when compared to sows with front cracked hooves.

The likelihood of rear digital overgrowth differed ( $\mathrm{P}<$ 0.01 ) between parities with older sows generally having a greater frequency among the cull sows evaluated. Rear digital overgrowth varied from $8.3 \%$ in parity 1 to $52.3 \%$ in parity 10 . The probability for cull sows having rear digital overgrowth was different $(\mathrm{P}<0.01)$ between culling codes. Sows culled for $\mathrm{BC}, \mathrm{G}$ and $\mathrm{O}$ had a greater $(\mathrm{P}<$ $0.05)$ incidence for rear digital overgrowth when compared to sows culled for $\mathrm{P}$ and $\mathrm{R}(35.7 \%, 35.7 \%$ and $33.9 \%$ vs. $18.6 \%$ and $15.8 \%$, respectively). Cull sows without rear digital overgrowth in comparison to those with had more $(\mathrm{P}<0.05)$ pigs born alive in the last litter $(0.54)$ and a trend $(\mathrm{P}=0.06)$ for increased pigs per sow per year (0.80).

\subsection{Shoulder Lesions}

The presence of shoulder lesions on cull sows differed $(\mathrm{P}<$ $0.01)$ between parities with older parities generally having a greater occurrence. Cull sows with shoulder 
Table 1. Physical characteristics and production LS MEANS by parity for 923 cull sows ${ }^{\ddagger}$ evaluated at two U.S. harvest facilities.

\begin{tabular}{|c|c|c|c|c|c|c|c|c|c|c|c|}
\hline & \multicolumn{11}{|c|}{ Parity } \\
\hline & 1 & 2 & 3 & 4 & 5 & 6 & 7 & 8 & 9 & 10 & \\
\hline Trait & $n=124$ & $n=74$ & $n=74$ & $n=71$ & $n=106$ & $n=113$ & $n=101$ & $n=100$ & $n=93$ & $n=67$ & P-value \\
\hline \multicolumn{12}{|l|}{ Front feet } \\
\hline Heel lesions, $\%$ & $24.4^{\mathrm{b}}$ & $11.0^{\mathrm{a}}$ & $17.6^{\mathrm{ab}}$ & $24.3^{\mathrm{bc}}$ & $37.6^{\mathrm{cd}}$ & $43.8^{\mathrm{d}}$ & $41.0^{\mathrm{d}}$ & $44.4^{\mathrm{d}}$ & $48.8^{\mathrm{d}}$ & $47.0^{\mathrm{d}}$ & 0.01 \\
\hline Cracked hooves, $\%$ & $17.9^{\mathrm{ab}}$ & $36.6^{\mathrm{c}}$ & $32.4^{\mathrm{c}}$ & $27.1^{\mathrm{bc}}$ & $12.9^{\mathrm{a}}$ & $18.8^{\mathrm{ab}}$ & $17.0^{\mathrm{ab}}$ & $13.1^{\mathrm{ab}}$ & $16.3^{\mathrm{ab}}$ & $15.2^{\mathrm{ab}}$ & 0.01 \\
\hline Digital overgrowth, \% & 0.0 & 0.0 & 2.7 & 1.4 & 1.0 & 1.8 & 3.0 & 5.1 & 9.8 & 6.1 & 0.19 \\
\hline \multicolumn{12}{|l|}{ Rear feet } \\
\hline Heel lesions, \% & $46.7^{\mathrm{a}}$ & $57.5^{\mathrm{ab}}$ & $67.6^{\mathrm{bc}}$ & $67.7^{\mathrm{bc}}$ & $72.6^{\mathrm{c}}$ & $70.4^{\mathrm{bc}}$ & $75.8^{\mathrm{c}}$ & $67.4^{\mathrm{bc}}$ & $74.4^{\mathrm{c}}$ & $56.9^{\mathrm{ab}}$ & 0.01 \\
\hline Digital overgrowth, \% & $8.3^{\mathrm{a}}$ & $9.6^{\mathrm{a}}$ & $12.7^{\mathrm{a}}$ & $27.9^{\mathrm{b}}$ & $27.5^{\mathrm{b}}$ & $30.6^{\mathrm{b}}$ & $33.3^{\mathrm{b}}$ & $32.7^{\mathrm{b}}$ & $40.0^{\mathrm{bc}}$ & $52.3^{\mathrm{c}}$ & 0.01 \\
\hline Cracked hooves, $\%$ & 9.2 & 20.6 & 11.3 & 20.6 & 17.7 & 17.6 & 12.1 & 10.2 & 18.9 & 7.7 & 0.10 \\
\hline Missing dewclaws, $\%$ & 1.7 & 2.7 & 2.8 & 10.3 & 5.9 & 7.4 & 4.0 & 5.1 & 12.2 & 7.7 & 0.07 \\
\hline Abscesses, $\%$ & 0.8 & 0.0 & 8.5 & 5.9 & 1.0 & 2.8 & 2.0 & 3.0 & 3.3 & 0.0 & 0.32 \\
\hline \multicolumn{12}{|l|}{ Shoulder lesions } \\
\hline None, $\%$ & $90.3^{\mathrm{bc}}$ & $97.3^{\mathrm{c}}$ & $86.5^{\mathrm{ab}}$ & $80.3^{\mathrm{a}}$ & $86.8^{\mathrm{ab}}$ & $80.5^{\mathrm{a}}$ & $86.2^{\mathrm{ab}}$ & $83.0^{\mathrm{ab}}$ & $85.0^{\mathrm{ab}}$ & $74.6^{\mathrm{a}}$ & 0.04 \\
\hline Abrasions, $\%$ & 8.1 & 0.0 & 10.8 & 15.5 & 8.5 & 15.0 & 10.0 & 12.0 & 8.6 & 22.4 & 0.17 \\
\hline Open, $\%$ & 1.6 & 2.7 & 2.7 & 4.2 & 4.7 & 3.5 & 4.0 & 5.0 & 5.4 & 1.5 & 0.90 \\
\hline \multicolumn{12}{|l|}{ Ovaries } \\
\hline Normal, \% & 85.3 & 90.4 & 82.2 & 84.3 & 84.6 & 78.2 & 88.0 & 86.9 & 89.9 & 85.1 & 0.48 \\
\hline Acyclic, $\%$ & 13.1 & 4.1 & 11.0 & 10.0 & 7.7 & 7.3 & 5.0 & 5.1 & 5.6 & 6.0 & 0.33 \\
\hline Cystic, \% & 1.6 & 5.5 & 6.9 & 5.7 & 7.7 & 14.6 & 7.0 & 8.1 & 4.5 & 9.0 & 0.11 \\
\hline \multicolumn{12}{|l|}{ Pregnancy } \\
\hline Pregnant, \% & $1.6^{\mathrm{a}}$ & $9.6^{\mathrm{bc}}$ & $4.1^{\mathrm{abc}}$ & $5.7^{\mathrm{abc}}$ & $6.7^{\mathrm{abc}}$ & $4.5^{\mathrm{abc}}$ & $12.0^{\mathrm{c}}$ & $12.1^{\mathrm{c}}$ & $2.3^{\mathrm{ab}}$ & $3.0^{\mathrm{ab}}$ & 0.03 \\
\hline \multicolumn{12}{|l|}{ Systemic lesions } \\
\hline Lung involvement $1 \%-10 \%{ }^{\mathrm{k}}$ & 0.8 & 1.4 & 9.5 & 8.5 & 2.8 & 5.3 & 8.9 & 7.0 & 6.5 & 12.0 & 0.10 \\
\hline Lung involvement $>10 \%{ }^{1}$ & 6.5 & 1.4 & 1.4 & 4.2 & 5.7 & 6.2 & 8.9 & 4.0 & 1.1 & 3.0 & 0.29 \\
\hline Pleural adhesions, $\%$ & 5.7 & 5.5 & 5.4 & 5.7 & 7.7 & 1.8 & 5.0 & 4.0 & 4.5 & 10.5 & 0.62 \\
\hline \multicolumn{12}{|l|}{ Teeth wear } \\
\hline Minimum, \% & $63.6^{\mathrm{c}}$ & $21.6^{\mathrm{b}}$ & $7.4^{\mathrm{a}}$ & $3.0^{\mathrm{a}}$ & $0.0^{\mathrm{a}}$ & $2.0^{\mathrm{a}}$ & $1.0^{\mathrm{a}}$ & $1.0^{\mathrm{a}}$ & $0.0^{\mathrm{a}}$ & $0.0^{\mathrm{a}}$ & 0.01 \\
\hline Moderate, \% & $30.5^{\mathrm{a}}$ & $64.9^{\text {cd }}$ & $75.0^{\mathrm{d}}$ & $52.9^{\mathrm{bcd}}$ & $57.5^{\mathrm{cd}}$ & $47.0^{\mathrm{bc}}$ & $42.4^{\mathrm{ab}}$ & $40.2^{\mathrm{ab}}$ & $36.1^{\mathrm{ab}}$ & $23.8^{\mathrm{a}}$ & 0.01 \\
\hline Severe, $\%$ & $6.0^{\mathrm{a}}$ & $13.5^{\mathrm{b}}$ & $17.7^{\mathrm{b}}$ & $44.1^{\text {cd }}$ & $42.6^{\mathrm{c}}$ & $51.0^{\mathrm{cd}}$ & $56.6^{\mathrm{de}}$ & $58.7^{\mathrm{de}}$ & $64.0^{\mathrm{de}}$ & $76.2^{\mathrm{e}}$ & 0.01 \\
\hline \multicolumn{12}{|l|}{ Teeth no. } \\
\hline Top teeth, no. & $20.9^{\mathrm{a}}$ & $21.0^{\mathrm{a}}$ & $21.8^{\mathrm{b}}$ & $21.7^{\mathrm{b}}$ & $21.9^{\mathrm{b}}$ & $21.8^{\mathrm{b}}$ & $21.8^{\mathrm{b}}$ & $21.8^{\mathrm{b}}$ & $21.8^{\mathrm{b}}$ & $21.8^{\mathrm{b}}$ & 0.01 \\
\hline Bottom teeth, no. & $21.1^{\mathrm{a}}$ & $21.7^{\mathrm{b}}$ & $22.0^{\mathrm{bc}}$ & $22.2^{\mathrm{c}}$ & $22.1^{\mathrm{c}}$ & $21.9^{\mathrm{bc}}$ & $22.1^{\mathrm{c}}$ & $22.0^{\mathrm{c}}$ & $22.1^{\mathrm{c}}$ & $22.1^{\mathrm{c}}$ & 0.01 \\
\hline \multicolumn{12}{|l|}{ Production } \\
\hline Lifetime pigs born alive & $9.9^{\mathrm{a}}$ & $20.5^{\mathrm{b}}$ & $33.8^{\mathrm{c}}$ & $44.9^{\mathrm{d}}$ & $55.4^{\mathrm{e}}$ & $64.7^{\mathrm{f}}$ & $74.7^{\mathrm{g}}$ & $86.8^{\mathrm{h}}$ & $97.2^{\mathrm{i}}$ & $108.8^{\mathrm{j}}$ & 0.01 \\
\hline Pigs per litter & $11.1^{\mathrm{bc}}$ & $10.6^{\mathrm{ab}}$ & $11.3^{\mathrm{c}}$ & $11.1^{\mathrm{bc}}$ & $11.0^{\mathrm{bc}}$ & $10.7^{\mathrm{abc}}$ & $10.6^{\mathrm{ab}}$ & $10.8^{\mathrm{abc}}$ & $10.6^{\mathrm{ab}}$ & $10.2^{\mathrm{a}}$ & 0.05 \\
\hline Pigs born alive in the last litter & $10.5^{\mathrm{cd}}$ & $11.1^{\mathrm{de}}$ & $11.0^{\text {de }}$ & $11.8^{\mathrm{e}}$ & $10.4^{\text {cd }}$ & $9.7^{\mathrm{bc}}$ & $9.9^{\mathrm{bc}}$ & $9.2^{\mathrm{ab}}$ & $9.2^{\mathrm{ab}}$ & $8.3^{\mathrm{a}}$ & 0.01 \\
\hline Non-productive days per parity & $47.6^{\mathrm{ef}}$ & $51.2^{\mathrm{f}}$ & $44.9^{\mathrm{e}}$ & $38.4^{\mathrm{d}}$ & $38.6^{\mathrm{d}}$ & $33.9^{\mathrm{c}}$ & $31.5^{\mathrm{bc}}$ & $30.0^{\mathrm{ab}}$ & $27.2^{\mathrm{a}}$ & $27.9^{\mathrm{ab}}$ & 0.01 \\
\hline Weaning to culling interval, $\mathrm{d}$ & $38.6^{\mathrm{c}}$ & $48.2^{\mathrm{de}}$ & $50.0^{\mathrm{e}}$ & $40.6^{\text {cde }}$ & $40.0^{\text {cd }}$ & $26.5^{\mathrm{b}}$ & $22.7^{\mathrm{b}}$ & $21.8^{\mathrm{b}}$ & $9.5^{\mathrm{a}}$ & $11.4^{\mathrm{a}}$ & 0.01 \\
\hline Pigs per sow per year & $24.3^{\mathrm{a}}$ & $23.9^{\mathrm{a}}$ & $26.2^{\mathrm{b}}$ & $26.9^{\mathrm{b}}$ & $26.6^{\mathrm{b}}$ & $26.7^{\mathrm{b}}$ & $26.8^{\mathrm{b}}$ & $27.3^{b}$ & $27.5^{\mathrm{b}}$ & $26.5^{\mathrm{b}}$ & 0.01 \\
\hline
\end{tabular}

"Sows were from eight farms within one integrated US pork production system and harvested at two midwestern sow harvest facilities. ${ }^{\text {abcdefghij }}{ }^{\text {Row means with }}$ different subscripts differ $(\mathrm{P}<0.05)$. ${ }^{\mathrm{k}}$ Sows with lesions involving $1 \%-10 \%$ of their lungs. ${ }^{\mathrm{I}}$ Sows with lesions involving $>10 \%$ of their lungs. 
Table 2. Physical characteristics and production LS MEANS by culling code for 923 cull sows ${ }^{\ddagger}$ evaluated at two US midwestern harvest facilities.

\begin{tabular}{|c|c|c|c|c|c|c|c|}
\hline & \multicolumn{6}{|c|}{ Culling Code $\mathrm{e}^{\mathrm{e}}$} & \\
\hline & $\mathrm{BC}$ & G & $\mathrm{L}$ & $\mathrm{O}$ & $\mathrm{P}$ & $\mathrm{R}$ & \\
\hline Trait & $n=90$ & $n=322$ & $n=83$ & $n=60$ & $n=73$ & $n=295$ & P-value \\
\hline \multicolumn{8}{|l|}{ Front feet } \\
\hline Heel lesions, \% & $31.8^{\mathrm{bc}}$ & $49.2^{\mathrm{d}}$ & $29.6^{\mathrm{bc}}$ & $15.0^{\mathrm{a}}$ & $41.4^{\mathrm{cd}}$ & $23.3^{\mathrm{ab}}$ & 0.01 \\
\hline Cracked hooves, $\%$ & $25.0^{\mathrm{bc}}$ & $14.4^{\mathrm{a}}$ & $30.9^{c}$ & $15.0^{\mathrm{ab}}$ & $15.7^{\mathrm{ab}}$ & $22.9^{\mathrm{bc}}$ & 0.01 \\
\hline Digital overgrowth, \% & 4.5 & 4.7 & 1.2 & 1.7 & 1.4 & 1.7 & 0.24 \\
\hline \multicolumn{8}{|l|}{ Rear feet } \\
\hline Heel lesions, \% & 64.3 & 70.4 & 57.9 & 59.3 & 65.7 & 64.3 & 0.26 \\
\hline Digital overgrowth, \% & $35.7^{\mathrm{c}}$ & $35.7^{\mathrm{c}}$ & $26.3^{\mathrm{bc}}$ & $33.9^{\mathrm{c}}$ & $18.6^{\mathrm{ab}}$ & $15.8^{\mathrm{a}}$ & 0.01 \\
\hline Cracked hooves, $\%$ & 16.7 & 12.4 & 18.4 & 11.9 & 11.4 & 16.2 & 0.57 \\
\hline Missing dewclaws, $\%$ & 7.1 & 5.7 & 5.3 & 11.9 & 4.3 & 4.8 & 0.44 \\
\hline Abscesses, $\%$ & 3.6 & 1.6 & 6.6 & 1.7 & 2.9 & 2.4 & 0.31 \\
\hline \multicolumn{8}{|l|}{ Shoulder lesions } \\
\hline None, $\%$ & $73.3^{\mathrm{a}}$ & $82.6^{\mathrm{b}}$ & $83.1^{\mathrm{ab}}$ & $85.0^{\mathrm{ab}}$ & $79.5^{\mathrm{ab}}$ & $93.9^{\mathrm{c}}$ & 0.01 \\
\hline Abrasions, $\%$ & $16.7^{\mathrm{b}}$ & $12.7^{\mathrm{b}}$ & $8.4^{\mathrm{ab}}$ & $15.0^{\mathrm{b}}$ & $17.8^{\mathrm{b}}$ & $5.1^{\mathrm{a}}$ & 0.01 \\
\hline Open, \% & $10.0^{\mathrm{c}}$ & $4.0^{\mathrm{b}}$ & $7.2^{\mathrm{bc}}$ & $0.0^{\mathrm{a}}$ & $2.7^{\mathrm{abc}}$ & $1.0^{\mathrm{a}}$ & 0.01 \\
\hline \multicolumn{8}{|l|}{ Ovaries } \\
\hline Normal, \% & 83.0 & 87.1 & 81.5 & 85.0 & 81.9 & 86.2 & 0.71 \\
\hline Acyclic, $\%$ & 10.2 & 6.0 & 9.9 & 10.0 & 11.1 & 6.6 & 0.45 \\
\hline Cystic, \% & 6.8 & 6.9 & 8.6 & 5.0 & 6.9 & 7.3 & 0.98 \\
\hline \multicolumn{8}{|l|}{ Pregnancy } \\
\hline Pregnant, \% & $6.7^{\mathrm{a}}$ & $4.1^{\mathrm{a}}$ & $4.9^{\mathrm{a}}$ & $28.3^{\mathrm{b}}$ & $4.2^{\mathrm{a}}$ & $4.5^{\mathrm{a}}$ & 0.01 \\
\hline \multicolumn{8}{|l|}{ Systemic lesions } \\
\hline Lung involvement $1 \%-10 \%{ }^{\mathrm{f}}$ & 9.8 & 12.9 & 11.2 & 5.8 & 10.5 & 6.8 & 0.49 \\
\hline Lung involvement $>10 \%{ }^{\mathrm{g}}$ & 5.6 & 5.3 & 8.4 & 3.3 & 6.8 & 2.0 & 0.15 \\
\hline Pleural adhesions & 5.6 & 5.4 & 7.3 & 8.3 & 4.2 & 4.5 & 0.81 \\
\hline \multicolumn{8}{|l|}{ Teeth wear } \\
\hline Minimum, \% & $7.1^{\mathrm{b}}$ & $0.4^{\mathrm{a}}$ & $9.7^{\mathrm{bc}}$ & $8.6^{\mathrm{bc}}$ & $9.4^{\mathrm{bc}}$ & $14.1^{\mathrm{c}}$ & 0.01 \\
\hline Moderate, $\%$ & 47.9 & 36.4 & 46.3 & 48.0 & 37.6 & 42.0 & 0.27 \\
\hline Severe, $\%$ & $41.2^{\mathrm{a}}$ & $63.6^{\mathrm{b}}$ & $37.5^{\mathrm{a}}$ & $40.3^{\mathrm{a}}$ & $48.0^{\mathrm{a}}$ & $35.1^{\mathrm{a}}$ & 0.01 \\
\hline \multicolumn{8}{|l|}{ Teeth wear } \\
\hline Top teeth, no. & $21.5^{\mathrm{ab}}$ & $21.7^{\mathrm{b}}$ & $21.6^{\mathrm{ab}}$ & $21.4^{\mathrm{a}}$ & $21.6^{\mathrm{ab}}$ & $21.5^{\mathrm{a}}$ & 0.05 \\
\hline Bottom teeth, no. & $21.8^{\mathrm{ab}}$ & $22.1^{\mathrm{b}}$ & $22.1^{\mathrm{b}}$ & $21.7^{\mathrm{a}}$ & $21.8^{\mathrm{ab}}$ & $21.7^{\mathrm{a}}$ & 0.01 \\
\hline \multicolumn{8}{|l|}{ Production } \\
\hline Average parity & $4.8^{c}$ & $8.0^{\mathrm{d}}$ & $4.1^{\mathrm{b}}$ & $4.5^{\mathrm{bc}}$ & $5.0^{\mathrm{c}}$ & $3.5^{\mathrm{a}}$ & 0.01 \\
\hline Lifetime pigs born alive & $47.4^{\mathrm{b}}$ & $86.5^{\mathrm{c}}$ & $44.3^{\mathrm{ab}}$ & $48.4^{\mathrm{b}}$ & $49.8^{\mathrm{b}}$ & $38.8^{\mathrm{a}}$ & 0.01 \\
\hline Pigs per litter & $11.2^{\mathrm{bc}}$ & $10.7^{\mathrm{ab}}$ & $11.0^{\mathrm{bc}}$ & $10.6^{\mathrm{abc}}$ & $10.3^{\mathrm{a}}$ & $11.0^{\mathrm{c}}$ & 0.02 \\
\hline Pigs born alive in last litter & $10.6^{\mathrm{c}}$ & $9.5^{\mathrm{b}}$ & $10.8^{\mathrm{c}}$ & $10.3^{\mathrm{bc}}$ & $8.4^{\mathrm{a}}$ & $10.7^{\mathrm{c}}$ & 0.01 \\
\hline Non-productive days per parity & $32.4^{\mathrm{ab}}$ & $30.5^{\mathrm{a}}$ & $35.0^{\mathrm{b}}$ & $40.7^{\mathrm{c}}$ & $31.6^{\mathrm{ab}}$ & $48.5^{\mathrm{d}}$ & 0.01 \\
\hline Weaning to culling interval, $\mathrm{d}$ & $22.8^{\mathrm{bc}}$ & $15.3^{\mathrm{a}}$ & $25.1^{\mathrm{c}}$ & $42.9^{\mathrm{d}}$ & $15.2^{\mathrm{ab}}$ & $57.9^{\mathrm{e}}$ & 0.01 \\
\hline Pigs per sow per year & $28.2^{\mathrm{c}}$ & $27.1^{\mathrm{bc}}$ & $26.8^{\mathrm{bc}}$ & $24.8^{\mathrm{a}}$ & $25.9^{\mathrm{ab}}$ & $24.8^{\mathrm{a}}$ & 0.01 \\
\hline
\end{tabular}

†Sows were from eight farms within one integrated US pork production system and harvested at two midwestern sow harvest facilities. ${ }^{\text {abcd }}$ Row means with different subscripts differ $(\mathrm{P}<0.05)$. ${ }^{\mathrm{e}} \mathrm{BC}=$ body condition, $\mathrm{G}=$ old age, $\mathrm{L}=$ lameness, $\mathrm{O}=$ other, $\mathrm{P}=$ poor litter performance, $\mathrm{R}=$ reproductive failure. ${ }^{\mathrm{f}}$ Sows with lesions involving $1 \%-10 \%$ of their lungs. ${ }^{\mathrm{g}}$ Sows with lesions involving $>10 \%$ of their lungs. 
Table 3. Physical characteristic contrasts (binary traits ${ }^{\dagger}$ ) and solution estimates (continuous traits ${ }^{\ddagger}$ ) for sow productivity traits $^{\text {Il }}$ on 923 cull sows ${ }^{\ddagger}$ evaluated at two US midwestern harvest facilities.

\begin{tabular}{|c|c|c|c|c|c|c|c|c|}
\hline \multirow[b]{2}{*}{ Trait } & \multicolumn{2}{|c|}{ LPBA } & \multicolumn{2}{|c|}{ PBALL } & \multicolumn{2}{|c|}{ PSY } & \multicolumn{2}{|c|}{ WCI } \\
\hline & $\begin{array}{c}\text { Absence - } \\
\text { presence }\end{array}$ & P-value & $\begin{array}{c}\text { Absence - } \\
\text { presence }\end{array}$ & P-value & $\begin{array}{c}\text { Absence - } \\
\text { presence }\end{array}$ & P-value & $\begin{array}{c}\text { Absence - } \\
\text { presence }\end{array}$ & P-value \\
\hline \multicolumn{9}{|l|}{ Front feet } \\
\hline Heel lesions ${ }^{\dagger}$ & -0.45 & 0.55 & 0.18 & 0.42 & -0.51 & 0.19 & 9.9 & 0.01 \\
\hline Cracked hooves $^{\dagger}$ & 0.50 & 0.57 & 0.00 & 0.98 & 0.80 & 0.07 & -5.4 & 0.05 \\
\hline Digital overgrowth $^{\dagger}$ & 0.22 & 0.92 & 0.35 & 0.55 & -0.07 & 0.94 & 0.8 & 0.89 \\
\hline \multicolumn{9}{|l|}{ Rear feet } \\
\hline Heel lesions ${ }^{\dagger}$ & -0.66 & 0.39 & -0.26 & 0.23 & 0.22 & 0.55 & -1.3 & 0.58 \\
\hline Digital overgrowth $^{\dagger}$ & 0.94 & 0.27 & 0.54 & 0.03 & 0.80 & 0.06 & -1.1 & 0.67 \\
\hline Cracked hooves ${ }^{\dagger}$ & 0.26 & 0.79 & -0.14 & 0.65 & -0.55 & 0.29 & 0.1 & 0.99 \\
\hline Missing dewclaws ${ }^{\dagger}$ & 2.15 & 0.16 & -0.02 & 0.97 & 0.40 & 0.60 & -6.4 & 0.16 \\
\hline Abscesses $^{\dagger}$ & -2.00 & 0.37 & -0.08 & 0.91 & -0.33 & 0.76 & 4.3 & 0.54 \\
\hline \multicolumn{9}{|l|}{ Shoulder lesions } \\
\hline None $^{\dagger}$ & -2.01 & 0.04 & -0.14 & 0.64 & -0.04 & 0.93 & -10.6 & 0.01 \\
\hline Abrasions ${ }^{\dagger}$ & 2.09 & 0.07 & 0.36 & 0.27 & 0.29 & 0.65 & 12.1 & 0.01 \\
\hline Open $^{\dagger}$ & 1.06 & 0.57 & -0.32 & 0.55 & -0.88 & 0.41 & 5.8 & 0.30 \\
\hline \multicolumn{9}{|l|}{ Ovaries } \\
\hline Normal $^{\dagger}$ & 0.68 & 0.50 & -0.27 & 0.36 & 0.11 & 0.82 & -5.6 & 0.06 \\
\hline Acyclic $^{\dagger}$ & -0.41 & 0.76 & 0.24 & 0.53 & -0.51 & 0.45 & 11.2 & 0.01 \\
\hline Cystic $^{\dagger}$ & -0.89 & 0.53 & 0.24 & 0.54 & 0.33 & 0.63 & -1.4 & 0.74 \\
\hline \multicolumn{9}{|l|}{ Pregnancy } \\
\hline Pregnant $^{\dagger}$ & -0.13 & 0.93 & 0.80 & 0.06 & 1.10 & 0.14 & -13.5 & 0.01 \\
\hline \multicolumn{9}{|l|}{ Systemic lesions } \\
\hline Lung $1 \%-10 \%{ }^{\dagger a}$ & -1.93 & 0.19 & -0.44 & 0.31 & -0.95 & 0.20 & 2.3 & 0.61 \\
\hline Lung $>10 \%{ }^{\dagger \mathrm{b}}$ & -2.49 & 0.13 & -0.45 & 0.36 & -1.20 & 0.14 & 9.6 & 0.06 \\
\hline Pleural adhesions $^{\dagger}$ & -1.58 & 0.30 & 0.13 & 0.77 & 0.26 & 0.74 & -1.8 & 0.70 \\
\hline \multicolumn{9}{|l|}{ Teeth wear } \\
\hline Minimum, $\%^{\dagger}$ & -1.00 & 0.50 & -0.24 & 0.58 & -1.10 & 0.15 & 11.6 & 0.01 \\
\hline Moderate, $\%^{\dagger}$ & -0.92 & 0.22 & -0.32 & 0.14 & -0.66 & 0.08 & 0.0 & 0.99 \\
\hline Severe, $\%^{\dagger}$ & 1.36 & 0.10 & 0.45 & 0.06 & 1.10 & 0.01 & -3.5 & 0.15 \\
\hline \multicolumn{9}{|l|}{ Teeth no. } \\
\hline Top teeth, no. ${ }^{\ddagger}$ & 0.20 & 0.61 & 0.17 & 0.13 & -0.14 & 0.50 & 3.64 & 0.01 \\
\hline Bottom teeth, no. ${ }^{*}$ & 0.07 & 0.82 & 0.04 & 0.67 & -0.08 & 0.61 & -0.51 & 0.59 \\
\hline $\mathrm{BCS}^{\ddagger \mathrm{c}}$ & -0.28 & 0.53 & 0.09 & 0.47 & -0.26 & 0.24 & 4.57 & 0.01 \\
\hline 10th rib backfat ${ }^{\ddagger}$ & -0.04 & 0.93 & -0.00 & 0.99 & -0.62 & 0.23 & 4.65 & 0.01 \\
\hline $\mathrm{LMA}^{\mathrm{d}}, \mathrm{cm}^{2 \ddagger}$ & -0.12 & 0.02 & -0.01 & 0.51 & -0.49 & 0.01 & 0.44 & 0.01 \\
\hline $\mathrm{LMD}^{\mathrm{e}}, \mathrm{cm}^{\ddagger}$ & -1.02 & 0.04 & -0.08 & 0.57 & -1.54 & 0.01 & 3.26 & 0.03 \\
\hline Lung, $\%^{\ddagger f}$ & 0.06 & 0.09 & 0.01 & 0.46 & 0.03 & 0.06 & -0.26 & 0.01 \\
\hline
\end{tabular}

${ }^{7} \mathrm{LPBA}=$ Lifetime pigs born alive, PBALL = Pigs born alive in last litter, PSY $=$ Pigs per sow per year, WCI $=\mathrm{Weaning}$ to culling interval. ${ }^{\ddagger}$ Sows were from eight farms within one integrated U.S. pork production system and harvested at two Midwestern sow harvest facilities. ${ }^{a}$ Sows with lesions involving $1 \%$ - $10 \%$

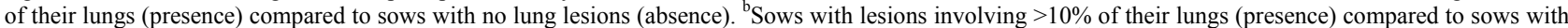
no lung lesions (absence). ${ }^{\mathrm{c}} \mathrm{BCS}=$ body condition score (possible range 1 to 5 , Patience and Thacker, 1989). ${ }^{\mathrm{d}} \mathrm{LMA}=\mathrm{Longissimus}$ muscle area. ${ }^{\mathrm{e}} \mathrm{LMD}=\mathrm{Long}-$ issimus muscle depth. ${ }^{\mathrm{f}}$ Total lung involvement from lesions. 
lesions ranged from $2.7 \%$ in parity 2 to $25.4 \%$ in parity 10 . The probability for the presence of shoulder lesions on cull sows was different $(\mathrm{P}<0.01)$ between culling codes. Sows culled for $\mathrm{R}$ had the lowest $(\mathrm{P}<0.05)$ likelihood for shoulder lesions when compared to the other five culling codes $(6.1 \%$ vs. $15.0 \%$ to $26.7 \%)$. Sows without shoulder lesions had greater $(\mathrm{P}<0.05)$ lifetime pigs born alive $(2.01)$ and averaged more $(\mathrm{P}<0.01)$ days from weaning to culling (10.6 d) when compared to sows having shoulder lesions.

The probability for the presence of open shoulder lesions on cull sows was different $(\mathrm{P}=0.01)$ between culling codes. Open shoulder lesions were greatest among sows culled for $\mathrm{BC}(10.0 \%)$ and $\mathrm{L}(7.2 \%)$.

\subsection{Reproductive Tracts}

Grossly normal appearing ovaries were not different $(\mathrm{P}>$ 0.05 ) between parities and culling codes among the cull sows evaluated in this study. The weaning to culling interval was shorter $(\mathrm{P}<0.01)$ for sows with acyclic ovaries when compared to those without (11.2 d).

The likelihood for pregnant females among cull sows evaluated differed $(\mathrm{P}<0.05)$ between parities and culling codes. There were more $(\mathrm{P}<0.01)$ pregnancies in the $\mathrm{O}$ culling code when compared to other 5 culling codes ( $28.3 \%$ vs. $4.1 \%$ to $6.7 \%)$. Sows that were observed pregnant at culling tended $(\mathrm{P}=0.06)$ to have fewer pigs born alive in their last litter $(0.80)$ when compared to cull sows that were not pregnant. Sows that were not pregnant at culling had fewer $(\mathrm{P}<0.01)$ days from weaning to culling when compared to cull sows that were found to be pregnant $(13.5 \mathrm{~d})$.

\subsection{Respiratory Systems}

The probability for cull sows with lesions involving $1 \%$ to $10 \%$ of the lungs, greater than $10 \%$ of the lungs or pleural adhesions did not differ between parities or culling codes. Sows with lesions that involved greater than $10 \%$ of the lung tissue tended $(\mathrm{P}=0.06)$ to have a shorter weaning to culling interval ( $9.6 \mathrm{~d}$ ) when compared to cull sows with no lung lesions. However, this difference was not observed $(\mathrm{P}>0.05)$ among cull sows where lung lesion involvement was from $1 \%$ to $10 \%$ when compared to sows without any lung lesion involvement. Regression coefficient estimates for percent lung lesion involvement were positive and tended $(\mathrm{P}<0.10)$ to be different from zero for lifetime pigs born alive (0.06) and pigs per sow per year $(0.03)$ and negative $(\mathrm{P}=0.01)$ for weaning to culling interval $(-0.26)$.

\subsection{Teeth Evaluation}

Minimum, moderate and severe teeth wear among the cull sows evaluated differed $(\mathrm{P}<0.01)$ among the pari- ties evaluated. Severe teeth wear increased as parity increased among the cull sows evaluated. Minimum teeth wear ranged from $63.6 \%$ in parity 1 to $0.0 \%$ in parity 9 and 10 . Severe teeth wear varied from $6.0 \%$ in parity 1 to $76.2 \%$ in parity 10 . Minimum and severe teeth wear were different across $(\mathrm{P}<0.01)$ culling codes. Cull sows removed for $\mathrm{G}$ had a lower percentage of sows with $(\mathrm{P}<$ $0.01)$ minimum teeth wear and greater percentage of sows having $(\mathrm{P}<0.05)$ severe teeth wear when compared to sows culled from the other 5 culling codes. Cull sows with moderate teeth wear tended $(\mathrm{P}=0.08)$ to have more pigs per sow per year (0.66) in comparison to those without moderate teeth wear. Sows with severe teeth wear tended $(\mathrm{P}<0.10)$ to have fewer lifetime pigs born alive (1.36), pigs born alive in last litter (0.45) and less $(\mathrm{P}<0.01)$ pigs per sow per year $(1.10)$ when compared to sows without severe teeth wear.

Top and bottom teeth number differed $(\mathrm{P}<0.01)$ between parities among the cull sows evaluated in this study. Cull sows from parities 1 and 2 had fewer $(\mathrm{P}<$ $0.01)$ top teeth and sows in parity 1 had fewer $(\mathrm{P}<0.01)$ bottom teeth when compared to cull sows from older parities.

\subsection{Body Condition and Composition}

Cull sow body composition LS MEANS by parity, culling code and body condition score are presented in Table 4. Backfat and BCS were not different $(\mathrm{P}>0.05)$ between parities among the cull sows evaluated in this study. However, BCS for the cull sows was different $(\mathrm{P}<$ 0.01 ) between parities within harvest plant. As parity increased, BCS from sows harvested at plant 1 tended to increase while BCS from sows harvested at plant 2 tended to decrease. Longissimus muscle area and depth from the cull sows were different $(\mathrm{P}<0.01)$ between parities. Longissimus muscle area generally increased from parity 1 to $5\left(44.8 \mathrm{~cm}^{2}\right.$ to $\left.47.8 \mathrm{~cm}^{2}\right)$ and then plateaued (Table 4).

Cull sow backfat, longissimus muscle area, longissimus muscle depth and BCS were different $(\mathrm{P}<0.01)$ between culling codes. Sows culled from the breeding herd for $\mathrm{BC}$ and $\mathrm{L}$ had less $(\mathrm{P}<0.01)$ backfat when compared to sows having other on-farm culling codes $(1.83 \mathrm{~cm}$ and $2.04 \mathrm{~cm}$ vs. $2.47 \mathrm{~cm}$ to $2.85 \mathrm{~cm}$, respectively). The $\mathrm{BC}$ culling code had the smallest $(\mathrm{P}<0.01)$ longissimus muscle area $\left(41.2 \mathrm{~cm}^{2}\right)$, longissimus muscle depth $(4.91 \mathrm{~cm})$ and lowest $(\mathrm{P}<0.05)$ body condition score (2.37). Sows culled for $\mathrm{L}$ had a lower $(\mathrm{P}<0.05)$ BCS than G, P or R sows. The R culling code had the greatest $(\mathrm{P}<0.01) \mathrm{BCS}$.

Backfat, longissimus muscle area and longissimus muscle depth were different $(\mathrm{P}<0.01)$ between body condition scores from the cull sows evaluated in this study. With each increase in body condition score, back- 


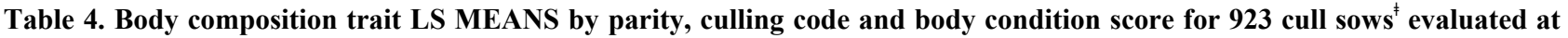
two US midwestern harvest facilities.

\begin{tabular}{|c|c|c|c|c|c|c|c|c|c|c|c|}
\hline \multicolumn{12}{|c|}{ Parity } \\
\hline Trait & 1 & 2 & 3 & 4 & 5 & 6 & 7 & 8 & 9 & 10 & P-value \\
\hline Backfat, cm & 2.61 & 2.54 & 2.65 & 2.22 & 2.56 & 2.59 & 2.53 & 2.35 & 2.65 & 2.63 & 0.21 \\
\hline $\mathrm{LMA}^{\mathrm{f}}, \mathrm{cm}^{2}$ & $44.8^{\mathrm{a}}$ & $45.3^{\mathrm{ab}}$ & $46.3^{\mathrm{abc}}$ & $45.6^{\mathrm{abc}}$ & $47.8^{\mathrm{bcd}}$ & $48.0^{\text {cd }}$ & $49.2^{\mathrm{d}}$ & $47.1^{\mathrm{bcd}}$ & $47.0^{\mathrm{abcd}}$ & $47.7^{\mathrm{bcd}}$ & 0.01 \\
\hline $\mathrm{LMD}^{\mathrm{g}}, \mathrm{cm}$ & $5.26^{\mathrm{a}}$ & $5.29^{\mathrm{ab}}$ & $5.30^{\mathrm{ab}}$ & $5.27^{\mathrm{ab}}$ & $5.53^{\mathrm{bc}}$ & $5.58^{\mathrm{c}}$ & $5.73^{\mathrm{c}}$ & $5.51^{\mathrm{bc}}$ & $5.53^{\mathrm{bc}}$ & $5.61^{\mathrm{c}}$ & 0.01 \\
\hline $\mathrm{BCS}^{\mathrm{h}}$ & 2.98 & 2.99 & 2.98 & 2.71 & 2.89 & 2.87 & 2.94 & 2.80 & 2.85 & 2.85 & 0.29 \\
\hline \multicolumn{12}{|c|}{ Culling Code ${ }^{\mathrm{i}}$} \\
\hline & $\mathrm{BC}$ & G & $\mathrm{L}$ & $\mathrm{O}$ & $\mathrm{P}$ & $\mathrm{R}$ & P-value & & & & \\
\hline Backfat, cm & $1.83^{\mathrm{a}}$ & $2.60^{\mathrm{b}}$ & $2.04^{\mathrm{a}}$ & $2.62^{\mathrm{bc}}$ & $2.47^{\mathrm{b}}$ & $2.85^{\mathrm{c}}$ & 0.01 & & & & \\
\hline LMA, $\mathrm{cm}^{2}$ & $41.2^{\mathrm{a}}$ & $47.2^{\mathrm{bc}}$ & $46.5^{\mathrm{b}}$ & $47.0^{\mathrm{bc}}$ & $46.3^{\mathrm{b}}$ & $48.8^{\mathrm{c}}$ & 0.01 & & & & \\
\hline $\mathrm{LMD}, \mathrm{cm}$ & $4.91^{\mathrm{a}}$ & $5.53^{\mathrm{bc}}$ & $5.37^{\mathrm{b}}$ & $5.47^{\mathrm{bc}}$ & $5.44^{\mathrm{bc}}$ & $5.60^{\mathrm{c}}$ & 0.01 & & & & \\
\hline $\mathrm{BCS}$ & $2.37^{\mathrm{a}}$ & $2.92^{\mathrm{c}}$ & $2.60^{\mathrm{b}}$ & $2.78^{\mathrm{bc}}$ & $2.86^{\mathrm{c}}$ & $3.09^{\mathrm{d}}$ & 0.01 & & & & \\
\hline \multicolumn{12}{|c|}{ Body Condition Score } \\
\hline & 1 & 2 & 3 & 4 & 5 & P-value & & & & & \\
\hline Backfat, cm & $1.07^{\mathrm{a}}$ & $1.76^{\mathrm{b}}$ & $2.71^{\mathrm{c}}$ & $3.64^{\mathrm{d}}$ & $4.68^{\mathrm{e}}$ & 0.01 & & & & & \\
\hline LMA, $\mathrm{cm}^{2}$ & $35.2^{\mathrm{a}}$ & $43.2^{\mathrm{b}}$ & $48.9^{\mathrm{c}}$ & $51.8^{\mathrm{d}}$ & $54.4^{\mathrm{d}}$ & 0.01 & & & & & \\
\hline $\mathrm{LMD}, \mathrm{cm}$ & $4.19^{\mathrm{a}}$ & $5.13^{\mathrm{b}}$ & $5.70^{\mathrm{c}}$ & $5.84^{\mathrm{d}}$ & $6.01^{\mathrm{cd}}$ & 0.01 & & & & & \\
\hline
\end{tabular}

${ }^{\text {t}}$ Sows were from eight farms within one integrated U.S. pork production system and harvested at two midwestern sow harvest facilities. ${ }^{\text {abcde }}$ Row means with different subscripts differ $(\mathrm{P}<0.05)$. ${ }^{\mathrm{f}} \mathrm{LMA}=$ Longissimus muscle area. ${ }^{\mathrm{g}} \mathrm{LMD}=$ Longissimus muscle depth. ${ }^{\mathrm{h}} \mathrm{BCS}=\mathrm{Body}$ condition score (possible range 1 to 5, Patience and Thacker, 1989). ${ }^{\mathrm{i}} \mathrm{BC}=$ body condition, $\mathrm{G}=$ old age, $\mathrm{L}=$ lameness, $\mathrm{O}=$ other, $\mathrm{P}=$ poor litter performance, $\mathrm{R}=$ reproductive failure.

fat increased $(\mathrm{P}<0.01)$. Longissimus muscle area increased $(\mathrm{P}<0.01)$ from body condition score 1 to 4 , but not 4 to $5(\mathrm{P}=0.19)$.

Regression coefficients for longissimus muscle area and depth were different $(\mathrm{P}<0.05)$ from zero for lifetime pigs born alive $(-0.12$ and -1.02 , respectively) and pigs per sow per year $(-0.49$ and -1.54 , respectively). The regression coefficients for $\mathrm{BCS}$, backfat, longissimus muscle area and longissimus muscle depth for weaning to culling interval were positive and different $(\mathrm{P}<0.05)$ from zero $(4.57,4.65,0.44$ and 3.26 , respectively).

\subsection{Production Data}

Lifetime pigs born alive, pigs per litter, pigs born alive in last litter, non-productive days per parity, weaning to culling interval, and pigs per sow per year differed $(\mathrm{P}<$ 0.05 ) between parities and culling codes from the cull sows evaluated in this study.

Lifetime pigs born alive increased $(\mathrm{P}<0.01)$ as parity increased. Among the sows evaluated, pigs per litter were lower $(\mathrm{P}<0.05)$ in parity 10 when compared to parities 1, 3, 4 and 5 (10.2 vs. 11.1, 11.3, 11.1, and 11.0, respectively). When evaluating the production records from the cull sows in the present study, pigs born alive in the last litter varied from 11.8 in parity 4 to 8.3 in parity 10. Non-productive days per parity generally decreased with increasing parity, ranging from $51.2 \mathrm{~d}$ in parity 2 to $27.2 \mathrm{~d}$ in parity 9. Pigs per sow per year was lower $(\mathrm{P}<$ 0.05 ) in parities 1 and 2 when compared to other parities (24.3 and 23.9 vs. 26.2 to 27.5 , respectively).

Sows culled for $\mathrm{G}$ had greater $(\mathrm{P}<0.01)$ lifetime pigs born alive when compared to the other 5 culling codes (86.5 vs. 38.8 to 49.8 , respectively). Sows culled for $\mathrm{R}$ tended $(\mathrm{P}<0.10)$ to have the fewest lifetime pigs born alive (38.8). Females culled for $\mathrm{P}$ had the fewest $(\mathrm{P}<$ $0.01)$ pigs born alive in last litter (8.4) when compared to the other 5 culling codes. Sows culled for $\mathrm{G}$ had fewer $(\mathrm{P}<$ $0.01)$ pigs born alive in last litter when compared to the BC, L and R culling codes (9.5 vs. 10.6, 10.8 and 10.7, respectively). The $\mathrm{R}$ culling code had greater $(\mathrm{P}<0.01)$ non-productive days per parity when compared to sows from the other five culling codes ( $48.5 \mathrm{~d}$ vs. 30.5 to 40.7 d). Culling codes $\mathrm{O}$ and $\mathrm{R}$ had the fewest pigs per sow per year (24.8 and 24.8, respectively). However, assuming each sows' first farrowing was at one year of age and adding 60 days to herd life to account for gilt development, pigs per sow per year was greatest $(\mathrm{P}<0.01)$ in the $\mathrm{G}$ culling code $(25.8)$ and lowest $(\mathrm{P}<0.01)$ in the $\mathrm{R}$ cul- 
ling code (21.7).

Culling code information by parity from the sows evaluated in this study is presented in Table 5. Reproductive failure was the most common culling code in parities 1 to $5(66.1 \%, 58.1 \%, 52.7 \%, 39.4 \%$ and $37.7 \%$, respectively). Sows not detected in estrus (no heat) was the most frequent farm culling code in parity $1(41.1 \%)$ and did not conceive most common in parities 2 to 5 (39.2\%, 36.5\%, 25.4\%, and $27.4 \%$, respectively). Body condition was the second most frequent culling code in parities 1 to $3(11.3 \%, 12.2 \%$, and $16.2 \%$, respectively). In parities 6 to $10, \mathrm{G}$ was the most common culling code $(30.1 \%, 60.4 \%, 71.0 \%, 81.7 \%$ and $86.6 \%$, respectively).

Front missing dew claws, front feet abscesses, peritonitis and shoulder abscesses were found in 2/908 (0.2\%), $7 / 903(0.7 \%), 16 / 895(1.7 \%)$ and $3 / 920(0.3 \%)$ of sows, respectively. Due to the relatively low incidence rate in each of these conditions they were not further evaluated.

\section{Discussion}

\subsection{Feet Lesions}

The probability for front and rear heel lesions was associated with increased parity. These findings are in greement with Brooks et al. [10] who reported heel lesions increased with increasing sow age.

Multiple factors are thought to influence heel lesions. Gjein and Larssen [8] suggest foot lesions may increase as body weight increases in older parities. This is supported by Lindemann et al. [11] who reported foot lesions were positively correlated with body weight in nursery pigs. In the present study, front heel lesions were associated with fewer days from weaning to culling. These results are supported by Gjein and Larssen [12] who reported the proportion of claw lesions decreased from the first to second month after farrowing. This suggests that front heel lesions may resolve during gestation.
Perhaps floor quality influenced feet and leg injuries. A study by MAFF [13] revealed concrete floors that were too slippery or too rough caused injuries in gestating sows. With concrete that was too slippery, sows tended to show swollen tendons, whereas with rough concrete, sows often exhibited abrasions on the pressure points of the feet. The same study reported foot problems with concrete slats having rough edges or slats set too wide apart. Gjein and Larssen [8] observed wet floors with accumulation of manure were associated with increased heel lesions.

Early parity sows had a greater likelihood for the presence of front cracked hooves. This could be explained at least in part by biotin deficiency. Studies have shown corn-based diets to be a good source of biotin, but deficiencies contributing to cracked hooves have been demonstrated in studies utilizing diets based on cereal grains other than corn $[10,14]$. In the current study, corn based diets were fed indicating that any biotin deficiency would have had to occur because of low sow feed intake either in gestation, lactation or both. However, feed intake information was not available in the present study so it is not possible to determine whether a biotin deficiency occurred. Simmins and Brooks [14] suggest a young, immature sow may be more likely to enter biotin deficiency at certain times in the breeding cycle (such as lactation) and hence, more prone to cracked hooves.

Facilities are another factor that can affect cracked hooves. Although facilities were similar in the present study, different gestation housing and flooring systems have shown to result in cracked hooves differences among the breeding animals. On concrete, sows housed in stalls generally have fewer rear cracked hooves when compared to sows housed in loose housing systems [15]. Comparing sows housed in concrete stalls or in deep litter bedding, the latter has been shown to produce fewer foot lesions on sows housed in this manner [12].

Table 5. Culling code information by parity for 923 cull sows ${ }^{\dagger}$ evaluated at two US midwestern harvest facilities.

\begin{tabular}{cccccccccccc}
\hline \multicolumn{10}{c}{ Parity } \\
\hline \multicolumn{1}{c}{ Culling code } & 1 & 2 & 3 & 4 & 5 & 6 & 7 & 8 & 9 & 10 \\
\hline & $n=124$ & $n=74$ & $n=74$ & $n=71$ & $n=106$ & $n=113$ & $n=101$ & $n=100$ & $n=93$ & $n=67$ \\
Body condition, \% & $11.3(2)^{\mathrm{a}}$ & $12.2(2)$ & $16.2(2)$ & $14.1(3)$ & $8.5(6)$ & $10.6(4)$ & $5.9(4)$ & $9.0(2)$ & $4.3(3)$ & $7.5(2)$ \\
Old age, \% & $1.6(6)$ & $1.4(6)$ & $2.7(5)$ & $5.6(6)$ & $12.3(4)$ & $30.1(1)$ & $60.4(1)$ & $71.0(1)$ & $81.7(1)$ & $86.6(1)$ \\
Lameness, \% & $10.5(3)$ & $10.8(3)$ & $14.9(3)$ & $21.1(2)$ & $14.2(3)$ & $8.0(5)$ & $5.9(4)$ & $5.0(4)$ & $1.1(6)$ & $0.0(5)$ \\
Other, \% & $4.8(5)$ & $10.8(3)$ & $10.8(4)$ & $9.9(4)$ & $9.4(5)$ & $7.1(6)$ & $6.9(3)$ & $4.0(5)$ & $2.2(4)$ & $0.0(5)$ \\
Poor litter performance, \% & $5.7(4)$ & $6.8(5)$ & $2.7(5)$ & $9.9(4)$ & $17.9(2)$ & $21.2(3)$ & $3.0(6)$ & $2.0(6)$ & $2.2(4)$ & $3.0(3)$ \\
Reproductive failure, \% & $66.1(1)$ & $58.1(1)$ & $52.7(1)$ & $39.4(1)$ & $37.7(1)$ & $23.0(2)$ & $17.8(2)$ & $9.0(2)$ & $8.6(2)$ & $3.0(3)$ \\
\hline
\end{tabular}

${ }^{\ddagger}$ Sows were from eight farms within one integrated US pork production system and harvested at two midwestern sow harvest facilities. ${ }^{\mathrm{a}} \mathrm{Culling}$ code rank within parity. 
Increased front cracked hooves appeared to be associated with the L culling code in the present study. This association is supported by Simmins and Brooks [14] who observed infected side-wall cracks often led to lameness. Perhaps mitigating cracked hooves would help prevent lameness among breeding herd females.

The probability for the presence of front and rear digital overgrowth among cull sows increased as parity increased. It has been postulated that pastern angle decreases as the sow gets older [16], which may reduce hoof wear. Rear pastern angles are smaller than front pasterns [16] which may contribute to the greater incidence for rear digital overgrowth when compared to overgrown front hooves (or toes) in the present study. Flooring and nutrition have been reported to be associated with overgrown hooves. Newton et al. [17] and MAFF [13] reported that pigs housed on plastic slats had a greater overgrown toe incidence when compared to pigs housed on concrete slats. Nutritionally, Jørgensen and Sørensen [18] reported sows reared on higher feeding levels during gilt development experienced longer dew claws.

In the current study, cull sows without rear digital overgrowth had greater pigs born alive in their last litter and exhibited a trend for increased pigs per sow per year $(\mathrm{P}=0.06)$ when compared to sows with overgrown rear toes. The explanation may be that sows with rear digital overgrowth spend less time feeding in lactation and eat less feed [19] which can reduce subsequent reproductive performance. Decreased lactation feed intake is known to reduce subsequent litter size [20] and increase weaning-to-conception interval [21].

\subsection{Shoulder Lesions}

The probability for shoulder abrasions among cull sows generally increased as parity increased. In agreement with the present findings, Davies et al. [22] found shoulder lesion incidence increased as parity increased. In the current study, sows with shoulder abrasions tended $(\mathrm{P}=$ $0.07)$ to have fewer lifetime pigs born alive. In contrast, Davies et al. [22] found no association between shoulder lesions and total number born. In the present study, sows with shoulder abrasions averaged fewer days from weaning to culling than sows without shoulder abrasions. Davies et al. [22] reported that shoulder lesions developed during lactation and healed rapidly during the following gestation. The occurrence of shoulder lesions is believed to be a multifactorial event affecting post-parturient sows [22]. Environmental factors including poor body condition, reduced activity level, lameness, moist skin, soiling of the floor and flooring type have been observed to increase the risk of shoulder lesions $[5,22,23]$.

\subsection{Reproductive Tracts}

The likelihood that cull sows had normal appearing ovaries did not differ between culling codes. Sows culled for $\mathrm{R}$ had an $86.2 \%$ probability of having grossly normal ovaries. In contrast, Dalin et al. [24] and Einarsson et al. [25] reported that the percentage of sows with normal ovaries but were culled for reproductive reasons $(69.4 \%$ and $52.6 \%$, respectively) was lower than in the present study. The underlying reasons sows have normal appearing ovaries but are culled from the breeding herd for reproductive failure are unknown. It is possible that this occurrence can be explained by variation in breeding management or stockmanship [26], poor estrous symptoms [27], genetic lines [28], or other reasons.

When compared to the present study, Heinonen et al. [4] reported a greater prevalence of acyclic ovaries from cull sows (25.1\%). Einarsson et al. [25] and Dalin et al. [24] reported that sows culled for reproductive disorders had an acyclic ovary incidence of $24.7 \%$ and $17.0 \%$, respectively. These values are greater than the likelihood of acyclic ovaries from sows culled for reproductive reasons in the current study (6.6\%). In the present study, weaning to culling interval was shorter for sows with acyclic ovaries when compared to sows without acyclic ovaries. These results may be influenced by sows culled during or immediately after lactation. This is supported by Einarsson et al. [25] who reported $69 \%$ of sows harvested during weeks 1 to 3 of lactation and $97 \%$ of sows harvested day 0 or 1 post-weaning were acyclic.

Cull sows with cystic ovaries when harvested did not differ between parities in the present study. Castagna et al. [29] reported numerical, but not significant differences for cystic ovary presence between first litter and multiparous sows $(1.0 \%$ vs. $2.6 \%)$ in an on-farm study using a real-time transcutaneous ultrasound. The same study reported sows with cystic ovaries had greater returns to estrus following insemination $(34.0 \%$ vs. $7.7 \%)$ and lower farrowing rates $(52.2 \%$ vs. $90.0 \%)$ when compared to sows without cystic ovaries. Similarly, Waberski et al. [30] reported that a greater percentage of sows with cystic ovaries had failed to conceive following insemination ( $14.6 \%$ vs. $5.2 \%)$ and lower farrowing rate $(62.8 \%$ vs. $83.7 \%)$ when compared to sows without cystic ovaries. The same study reported sows with ovarian cysts or cyst-like structures were more likely to be culled $(9.6 \%$ vs. $2.9 \%)$ when compared to sows without ovarian cysts.

Cull sows that were pregnant at harvest tended to have fewer pigs born alive in their last litter when compared to sows that were not pregnant at harvest. Perhaps farm managers attempted to cull the least productive sows when exceeding breeding targets. However, there was no difference in lifetime pigs born alive between pregnant 
and non-pregnant sows in the present study. This suggests sows should not be culled based on single litter performance. Walker et al. [31] reported litter size has a low repeatability $(r=0.14)$ providing further evidence sows should not be cullied based on performance from one litter.

\subsection{Respiratory Systems}

In comparison to the present study, lung lesion presence in cull sows has been previously reported to be greater (21\%) by Ritter et al. [5] and even greater in market pigs $[32,33](75.0 \%$ and $79.5 \%$, respectively). In the present study, the presence of lesions on the lungs from cull sows tended to be associated with increased lifetime pigs born alive and pigs per sow per year. Therefore, high producing females appear to be more susceptible to lungs lesions. Sows with lung lesions (greater than 10\% lung involvement) may be identifiable as they were culled earlier post-weaning when compared to sows culled with no or few lung lesions. Knauer et al. [34] reported the presence of lung lesions was greater in cull sows with poor body condition. Thus maintaining proper sow body condition may mitigate the presence of lung lesions in high producing females.

\subsection{Teeth Evaluation}

Cull sows having severe teeth wear when compared to cull sows without teeth wear tended to have fewer pigs born alive in the litter prior to culling and had fewer pigs per sow per year. These results support the findings from Sekiguchi and Koketsu [35] who reported females with a high frequency of vacuum chewing during gestation produced fewer total number born (11.7 vs. 12.6) and tended to have fewer pigs born alive (10.6 vs. 11.3) when compared to those that did not vacuum chew. Perhaps the present results indicate severe teeth wear, when corrected for parity, is an indication of stress due its association with poor reproductive performance. Other studies have reported that stressors reduce reproductive performance [36,37]. High ambient temperatures have been reported to decrease embryonic survival after fertilization [36]. Hemsworth et al. [37] reported farms with timid sows were associated with lower total pigs per sow per year when compared to those farms without timid sows. In that study, the authors defined timid as the time sows took to resume feeding after hand contact from the experimenter.

Cull sows in parities 1 and 2 had fewer top teeth and cull sows in parity 1 had fewer bottom teeth when compared to sows that were culled at other parities. These results are supported by Pond and Mersmann [38] who report pigs' full permanent dentition is acquired when they are approximately 18 months of age (parity 1 and parity 2).

\subsection{Body Condition and Composition}

Body condition and backfat were not different between parities in the present study. In contrast, Gjein and Larssen [15] and Bonde et al. [23] reported older sows were in better body condition than younger sows. One difference between the two studies was that sows were housed in gestation stalls in the present study and in gestation pens in the Gjein and Larssen [15] and Bonde et al. [23] studies. Gestation stalls typically allow for greater individual sow feed management when compared to group gestation sow pens. It is possible that the relatively widespread gestation stall use in the present study explains the consistent sow body condition observed across parities.

Sows culled for $\mathrm{L}$ were leaner and had poorer body condition than $G, P$ and $R$ sows. These results are supported by Bonde et al. [23] who observed severely lame sows were often in poorer body condition. Serenius et al. [39] reported skeletal locomotion had a negative genetic correlation with lean percentage in Landrace $(-0.31)$ and Large White $(-0.24)$ gilts. This correlation suggests lean gilts may be predisposed to lameness. However, selecting gilts with good structural conformation, feeding sows to an appropriate body condition and providing them with good care are management strategies to mitigate locomotor or lameness problems. In the current study, R sows had the heaviest body condition. These sows likely gained body condition from additional days on feed from weaning until culling.

Both backfat and longissimus muscle area increased as BCS increased from the cull sows evaluated in the present study. However, backfat had a greater impact on distinguishing body condition scores 4 and 5 when compared to longissimus muscle area. Collectively these results suggest muscling explains more variation in thinner cull sows in comparison to overly fat cull sows.

Lifetime pigs born alive and pigs per sow per year increased as longissimus muscle area and depth at culling decreased among the cull sows evaluated in this study. Perhaps sows producing fewer lifetime pigs born alive and pigs per sow per year were able to maintain higher body protein stores due to reduced production. Tarrés et al. [40] reported maternal Duroc gilts that had loin depths greater than $5.0 \mathrm{~cm}$ at first farrowing tended to have poorer length of productive life than sows with less muscle. Holm et al. [41] reported negative genetic correlations between lean meat content in gilts and parity 1 and 2 number born alive $(-0.12$ and -0.24 , respectively) in the Norwegian Landrace sow population. In the present study, body condition score, backfat, longissimus muscle area and longissimus muscle depth increased as the number of days between weaning to culling interval 
increased for the cull sows evaluated. This suggests sows gained body condition from weaning their last litter until they were culled.

\subsection{Production Data}

Sows culled at parities 1, 3, 4 and 5 had more pigs per litter compared to sows culled at parity 10 . These results are in agreement with Rodriguez-Zas et al. [42]. Nonproductive days per parity and weaning to culling interval decreased as parity increased. These results are supported by Dagorn and Aumaitre [43] who found weaning to mating interval and weaning to culling interval decreased as parity increased. Pigs per sow per year was poorest in parities 1 and 2 . These results are supported by Lucia et al. [44] who reported pigs weaned per day per mated female increased as parity increased. Hence, the inefficiencies from sows culled in early parities appears costly to the production system.

Sows culled for $\mathrm{R}$ tended to have the fewest lifetime pigs born alive (38.8) when compared to other culling codes in the present study. These results are supported by Lucia et al. [45] who reported sows culled for reproductive failure had the lowest lifetime pigs born alive. The $\mathrm{R}$ culling code had the most non-productive days per parity (48.5 d) which is agreement with Lucia et al. [45].

Reproductive failure was the most common culling code in parities 1 to 5 among the sows culled in the present study. This is in agreement with several other studies where reproductive failure was the most frequently reported culling reason in early parities [45-47]. Within the reproductive failure category, females not showing estrus appears to be a larger problem among gilts than sows $[4,48]$ and younger than older sows $[46]$. The current study reported anestrous the most common reason parity 1 females were culled but in parities 2 to 5 sows the most common removal reason was did not conceive. Serenius et al. [49] reported first farrowing interval to be lowly heritable ( 0.10 to 0.11$)$ indicating the majority of variation in rebreeding is due to environmental factors. Identifying and correcting suboptimal farm specific fertility factors offers an opportunity to improve culling for reproductive failure and hence, sow lifetime productivity.

Old age was the most common culling code in parities 6 and greater. These results are in agreement with several other studies that reported old age was the most common culling code in higher parities [45-47]. When culling for productivity does not occur, the natural life span for swine has been estimated to be 12 to 15 years of age [38]. Sows culled younger than their natural life span should be culled for reproductive failure, poor litter performance, or some other productivity reason rather than being culled for old age in order to better understand the reasons high parity sows are removed from the breeding herd.

\section{Acknowledgements}

The authors acknowledge the financial support given by the Hatch Act, State of Iowa funds and by the National Pork Board, Project No. 04-127.

\section{REFERENCES}

[1] K. J. Stalder, M. Knauer, T. J. Baas, M. F. Rothschild and J. W. Mabry, "Sow Longevity," Pig News and Information, Vol. 25, No. 2, 2004, pp. 53-74.

[2] S. S. Anil and J. Deen, "Benchmark Pig CHAMP Year in Review," 2007.

http://www.pigchamp.com/Portals/_default/Skins/PigCha $\mathrm{mp} /$ Creative/Assets/PDF/Benchmark_2007_File_A.pdf

[3] J. Deen, "Benchmark 2007 Summary of the Pig CHAMP Database," 2008.

http://www.pigchamp.com/Portals/_default/Skins/PigCha $\mathrm{mp} /$ Creative/Assets/PDF/Benchmark_2008.pdf

[4] M. Heinonen, A. Leppävuori and S. Pyörälä, "Evaluation of Reproductive Failure of Female Pigs Based on Slaughterhouse Material and Herd Record Survey," Animal Reproduction Science, Vol. 52, No. 3, 1998, pp. 235-244. doi:10.1016/S0378-4320(98)00105-5

[5] L. A. Ritter, J. L. Xue, G. D. Dial, R. B. Morrison and W. E. Marsh, "Prevalence of Lesions and Body Condition Scores among Female Swine at Slaughter," Journal of the American Veterinary Medical Association, Vol. 214, No. 4, 1999, pp. 525-528.

[6] J. F. Patience and P. A. Thacker, "Swine Nutrition Guide," Prairie Swine Centre, Saskatoon, 1989.

[7] Subcommittee on Swine Nutrition, Committee on Animal Nutrition and National Research Council, "Nutrient Requirements for Swine," 10th Revised Edition, The National Academies Press, Washington DC, 1998.

[8] H. Gjein and R. B. Larssen, "Housing of Pregnant Sows in Loose and Confined Systems-A Field Study 2. Claw Lesions: Morphology, Prevalence, Location and Relation to Age," Acta Veterinaria Scandinavica, Vol. 36, No. 4, 1995, pp. 433-442.

[9] SAS Institute, "SAS User's Guide: Statistics, Release 9.1," SAS Institute, Inc., Cary, 2003.

[10] P. H. Brooks, D. A. Smith and V. C. R. Irwin, "BiotinSupplementation of Diets; the Incidence of Foot Lesions, and the Reproductive Performance of Sows," Veterinary Record, Vol. 101, No. 3, 1977, pp. 46-50. doi:10.1136/vr.101.3.46

[11] M. D. Lindemann, E. T. Kornegay and E. R. Collins Jr., "The Effect of Various Flooring Materials on Performance and Foot Health of Early-Weaned Pigs," Livestock Production Science, Vol. 13, No. 4, 1985, pp. 373-382. doi:10.1016/0301-6226(85)90028-4

[12] H. Gjein and R. B. Larssen, "Housing of Pregnant Sows in Loose and Confined Systems-A Field Study 3. The Impact of Housing Factors on Claw Lesions," Acta Veterinaria Scandinavica, Vol. 36, No. 4, 1995, pp. 443450.

[13] MAFF, "Injuries Caused by Flooring: A Survey in Pig 
Health Scheme Herds," Proceedings Pig Veterinary Society, Vol. 8, 1981, pp. 119-125.

[14] P. H. Simmins and P. H. Brooks, "Supplementary Biotin for Sows: Effect on Claw Integrity," Veterinary Record, Vol. 122, No. 18, 1988, pp. 431-435. doi:10.1136/vr.122.18.431

[15] H. Gjein and R. B. Larssen, "Housing of Pregnant Sows in Loose and Confined Systems-A Field Study 1. Vulva and Body Lesions, Culling Reasons and Production Results," Acta Veterinaria Scandinavica, Vol. 36, No. 2, 1995, pp. 185-200.

[16] R. A. Barczewski, E. T. Kornegay, D. R. Notter, H. P. Veit and M. E. Wright, "Effects of Feeding Restricted Energy and Elevated Calcium and Phosphorus during Growth on Gait Characteristics of Culled Sows and Those Surviving Three Parities," Journal of Animal Science, Vol. 68, No. 10, 1990, pp. 3046-3055.

[17] G. L. Newton, C. V. Booram, O. M. Hale and B. G. Mullinix Jr., "Effect of Four Types of Floor Slats on Certain Feet Characteristics and Performance of Swine," Journal of Animal Science, Vol. 50, No. 1, 1980, pp. 7-20.

[18] B. Jørgensen and M. T. Sørensen, "Different Rearing Intensities of Gilts: II. Effects on Subsequent Leg Weakness and Longevity," Livestock Production Science, Vol. 54, No. 2, 1998, pp. 167-171.

[19] R. F. Fitzgerald, K. J. Stalder, L. A. Karriker, L. J. Sadler, H. T. Hill, J. Kaisand and A. K. Johnson, "The Effect of Hoof Abnormalities on Sow Behavior and Performance," Livestock Science, Vol. 145, No. 1-3, 2012, pp. 230-238. doi:10.1016/j.livsci.2012.02.009

[20] Y. Koketsu, G. D. Dial, J. E. Pettigrew and V. L. King, "Influence of Feed Intake during Individual Weeks of Lactation on Reproductive Performance of Sows on Commercial Farms," Livestock Production Science, Vol. 49, No. 3, 1997, pp. 217-225. doi:10.1016/S0301-6226(97)00050-X

[21] Y. Koketsu, G. D. Dial, J. E. Pettigrew and V. L. King, "Feed Intake Pattern during Lactation and Subsequent Reproductive Performance of Sows," Journal of Animal Science, Vol. 74, No. 12, 1996, pp. 2875-2884.

[22] P. R. Davies, W. E. M. Morrow, W. G. Rountree and D. C. Miller, "Epidemiologic Evaluation of Decubital Ulcers in Farrowing Sows," Journal of the American Veterinary Medical Association, Vol. 210, No. 8, 1997, pp. 11731178.

[23] M. T. Bonde, M. T. Rousing, J. H. Badsberg and J. T. Sørensen, “Associations between Lying-Down Behaviour Problems and Body Condition, Limb Disorders and Skin Lesions of Lactating Sows Housed in Farrowing Stalls in Commercial Sow Herds," Livestock Production Science, Vol. 87, No. 2-3, 2004, pp. 179-187. doi:10.1016/j.livprodsci.2003.08.005

[24] A.-M. Dalin, K. Gidlund and L. Eliasson-Selling, "Post Mortem Examination of Genital Organs from Sows with Reproductive Disturbances in a Sow-Pool," Acta Veterinaria Scandinavica, Vol. 38, No. 3, 1997, pp. 253-262.

[25] S. Einarsson, N. Lundeheim, K. Martinsson, N. Persson and I. Persson, "Post Mortem Examination of the Genital Organs of Culling Sows from One Large Herd with Rela- tion to Fertility Data," Proceedings International Pig Veterinary Society, Mexico, 26-31 July 1982, p. 211.

[26] W. L. Flowers, "Management of Reproduction," In: J. Wiseman, M. A. Varley and J. P. Chadwick, Eds., Progress in Pig Science, Nottingham University Press, Nottingham, 1997, pp. 383-405.

[27] L. Rydhmer, L. Eliasson-Selling, K. Johansson, S. Stern and K. Andersson, "A Genetic Study of Estrus Symptoms at Puberty and Their Relationship to Growth and Leanness in Gilts," Journal of Animal Science, Vol. 72, No. 8, 1994, pp. 1964-1970.

[28] P. Tummaruk, N. Lundeheim, S. Einarsson and A.-M. Dalin, "Repeat Breeding and Subsequent Reproductive Performance in Swedish Landrace and Swedish Yorkshire Sows," Animal Reproduction Science, Vol. 67, No. 3, 2001, pp. 267-280. doi:10.1016/S0378-4320(01)00129-4

[29] C. D. Castagna, C. H. Peixoto, F. P. Bortolozzo, I. Wentz, G. B. Neto and F. Ruschel, "Ovarian Cysts and Their Consequences on the Reproductive Performance of Swine Herds," Animal Reproduction Science, Vol. 81, No. 1, 2004, pp. 115-123. doi:10.1016/j.anireprosci.2003.08.004

[30] D. Waberski, A. Kunz-Schmidt, G. Borchardt Neto, L. Richter and K. F. Weitze, "Real-Time Ultrasound Diagnosis of Ovulation and Ovarian Cysts in Sows and Its Impact on Artificial Insemination Efficiency," Proceedings of American Society of Animal Science, Indianapolis, June 12012. http://www.asas.org/jas/symposia/proceedings/0944.pdf

[31] I. J. Walker, R. G. Beilharz and A. C. Dunkin, "A Genetic Study of Reproductive Performance in a Large Commercial Pig Herd," Proceedings of Australian Society of Animal Production, Canberra, February 1972, pp. 147152.

[32] M. R. Wilson, R. Takov, R. M. Friendship, S. W. Martin, I. McMillan, R. R. Hacker and S. Swaminathan, "Prevalence of Respiratory Diseases and Their Association with Growth Rate and Space in Randomly Selected Swine Herds," Canadian Journal of Veterinary Research, Vol. 50, No. 2, 1986, pp. 209-216.

[33] A. B. Scheidt, V. B. Mayrose, M. A. Hill, L. K. Clark, T. R. Cline, K. E. Knox, L. J. Runnels, S. Frantz and M. E. Einstein, "Relationship of Growth Performance to Pneumonia and Atrophic Rhinitis Detected in Pigs at Slaughter," Journal of the American Veterinary Medical Association, Vol. 196, No. 6, 1990, pp. 881-884.

[34] M. Knauer, K. J. Stalder, L. Karriker, T. J. Baas, C. Johnson, T. Serenius, L. Layman and J. D. McKean, "A Descriptive Survey of Lesions from Cull Sows Harvested at Two Midwestern U.S. Facilities," Preventive Veterinary Medicine, Vol. 82, No. 3-4, 2007, pp. 198-212. doi:10.1016/j.prevetmed.2007.05.017

[35] T. Sekiguchi and Y. Koketsu, "Behavior and Reproductive Performance by Stalled Breeding Females on a Commercial Swine Farm," Journal of Animal Science, Vol. 82, No. 5, 2004, pp. 1482-1487.

[36] R. L. Edwards, I. T. Omtvedt, E. J. Turman, D. F. Stephens and G. W. A. Mahoney, "Reproductive Performance of Gilts Following Heat Stress Prior to Breeding and in Early Gestation," Journal of Animal Science, 
Vol. 27, No. 6, 1968, pp. 1634-1637.

[37] P. H. Hemsworth, A. Brand and P. Willems, "The Behavioural Response of Sows to the Presence of Human Beings and Its Relation to Productivity," Livestock Production Science, Vol. 8, No. 1, 1981, pp. 67-74. doi:10.1016/0301-6226(81)90031-2

[38] W. G. Pond and H. J. Mersmann, "Biology of the Domestic Pig," Cornell University Press, New York, 2001.

[39] T. Serenius, M.-L. Sevón-Aimonen and E. A. Mäntysaari, "The Genetics of Leg Weakness in Finnish Large White and Landrace populations," Livestock Production Science, Vol. 69, No. 2, 2001, pp. 101-111. doi:10.1016/S0301-6226(00)00260-8

[40] J. Tarrés, J. Tibau, J. Piedrafita, E. Fàbrega and J. Reixach, "Factors Affecting Longevity in Maternal Duroc Swine Lines," Livestock Science, Vol. 100, No. 2-3, 2006, pp. 121-131. doi:10.1016/j.livprodsci.2005.08.007

[41] B. Holm, M. Bakken, G. Klemetsdal and O. Vangen, "Genetic Correlations between Reproduction and Production Traits in Swine," Journal of Animal Science, Vol. 82, No. 12, 2004, pp. 3458-3464.

[42] S. L. Rodriguez-Zas, B. R. Southey, R. V. Knox, J. F. Connor, J. F. Lowe and B. J. Roskamp, "Bioeconomic Evaluation of Sow Longevity and Profitability," Journal of Animal Science, Vol. 81, No. 12, 2003, pp. 2915-2922.

[43] J. Dagorn and A. Aumaitre, "Sow Culling: Reasons for and Effect on Productivity," Livestock Production Science, Vol. 6, No. 2, 1979, pp. 167-177. doi:10.1016/0301-6226(79)90018-6
[44] T. Lucia, G. Dial and W. E. Marsh, "Lifetime Reproductive and Financial Performance of Female Swine," Journal of the American Veterinary Medical Association, Vol. 216, No. 11, 2000, pp. 1802-1809. doi:10.2460/javma.2000.216.1802

[45] T. Lucia, G. Dial and W. E. Marsh, "Lifetime Reproductive Performance in Female Pigs Having Distinct Reasons for Removal," Livestock Production Science, Vol. 63, No. 3, 2000, pp. 213-222. doi:10.1016/S0301-6226(99)00142-6

[46] A. A. Dijkhuizen, R. M. M. Krabbenborg and R. B. M. Huirne, "Sow Replacement: A Comparison of Farmers' Actual Decisions and Model Recommendations," Livestock Production Science, Vol. 23, No. 1-2, 1989, pp. 207-218. doi:10.1016/0301-6226(89)90015-8

[47] T. E. Stein, A. Dijkhuizen, S. D'Allaire and R. S. Morris, "Sow Culling and Mortality in Commercial Swine Breeding Herds," Preventive Veterinary Medicine, Vol. 9, No. 2, 1990, pp. 85-94. doi:10.1016/0167-5877(90)90027-F

[48] A. Lyczyński and W. Kaczmarek, "Causes of Early Discarding Gilts and Sows from Breeding Herd on the Example of a Productive Pig Farm in Denmark," Proceedings of the 14th International Pig Veterinary Society Congress, Bologna, 7-10 July 1996, p. 542.

[49] T. Serenius, M.-L. Sevón-Aimonen, E. A. Kause, E. A Mäntysaari and A. Mäki-Tanila, "Selection Potential of Different Prolificacy Traits in the Finnish Landrace and Large White Populations," Acta Veterinaria Scandinavica, Vol. 54, No. 1, 2004, pp. 36-43. 